\title{
A case of renal hypouricemia and a G774A gene mutation causing acute renal injury that was improved by hemodialysis
}

\author{
Shuma Hirashio - Kyoko Yamada • Takayuki Naito • \\ Takao Masaki
}

Received: 16 December 2011/ Accepted: 8 February 2012/Published online: 30 March 2012

(C) Japanese Society of Nephrology 2012

\begin{abstract}
A 16-year-old man came to our hospital complaining of loin pain after a middle-distance race. Following admission, his renal dysfunction worsened rapidly, requiring several hemodialysis sessions. A renal biopsy showed no change in the glomeruli, although interstitial edema was observed. Following the recovery of renal function, we confirmed that his uric acid level was abnormally low and urate clearance was abnormality high. Gene analysis showed that he had a G774A mutation which dominated the SLC22A12 gene encoding the urate transporter 1 .
\end{abstract}

Keywords Renal hypouricemia - Urate transporter 1 (URAT1) · Gene mutation (SLC22A12, G774A) · Acute renal failure with severe loin pain and patchy renal ischemia after anaerobic exercise (ALPE)

S. Hirashio $(\bowtie) \cdot K$. Yamada $\cdot$ T. Masaki

Department of Nephrology, Hiroshima University Hospital,

1-2-3 Kasumi, Minami-ku, Hiroshima 734-8551, Japan

e-mail: hirashio@hiroshima-u.ac.jp

K. Yamada

e-mail: rsxyd216@yahoo.co.jp

T. Masaki

e-mail: masakit@hiroshima-u.ac.jp

S. Hirashio

Department of Nephrology, National Hospital Organization Kure

Medical Center and Chugoku Cancer Center, Kure, Japan

T. Naito

Department of Nephrology, Hiroshima Prefectural Hospital,

Hiroshima, Japan

e-mail: t.naito@do.enjoy.ne.jp

\section{Introduction}

Renal hypouricemia causes acute kidney injury (AKI) following extensive anaerobic exercise. However, AKI caused by renal hypouricemia is usually mild and recovers spontaneously without the requirement for hemodialysis (HD) treatment.

Renal hypouricemia is a disorder characterized by increased urate clearance and a significant decrease in the serum uric acid (UA) level. Recently, the urate transporter in the human kidney (URAT1) was identified, with nonsense mutations in the SLC22A12 gene encoding URAT1 also being demonstrated [1]. It has been shown that patients with hypouricemia have this mutation. It is also known that anaerobic exercise often causes AKI in patients with hypouricemia. This type of AKI is called "Acute renal failure with severe Loin Pain and patchy renal ischemia after anaerobic Exercise (ALPE)" [2-4].

The gene mutation in the case described in this report was a homozygous transmutation of G774A. This is the most common gene abnormality of URAT1 found in Japanese subjects [5]. Recovery from ALPE due to hypouricemia does not usually require HD treatment. However, our case required $\mathrm{HD}$, as his clinical condition was consistent with a rapidly progressive glomerulonephritis (RPGN). We consider that the clinical course in our case was rare, with renal hypouricemia causing ALPE and AKI that required HD. In addition, a rare feature of our case was that a kidney biopsy was performed during the acute phase. This allowed the evaluation of the kidney tissue histology. In this paper, we report the interesting and rare features of this case.

\section{Case report}

A 16-year-old man was admitted with loin pain after a $5-\mathrm{km}$ middle-distance race. When he first came to our out- 
patient clinic, his serum creatinine (Cre) level was increased $(4.6 \mathrm{mg} / \mathrm{dL})$. In addition, urinary examination confirmed the presence of proteinuria $(1+)$ and microhematuria $(1+)$. Two days later, he was admitted to our hospital for study. The patient provided written, informed consent and the study protocol was approved by the local ethics committee of the National Hospital Organization of the Kure Medical Center and Chugoku Cancer Center and the Jikei University School of Medicine. These additional investigations showed that his renal dysfunction had deteriorated rapidly since admission, with a urea nitrogen (UN) of $90 \mathrm{mg} / \mathrm{dL}$ and Cre of $7.6 \mathrm{mg} / \mathrm{dL}$. These results and his clinical condition were consistent with an RPGN.

No urine abnormalities had been identified in school medical examinations and the patient had no previous medical history of note. Increases in myoglobin and creatine phosphokinase were not found (Table 1). Ultrasound studies and computed tomography (CT) (Fig. 1) showed slightly enlarged bilateral kidneys, a finding frequently associated with AKI. After hospitalization, his kidney function deteriorated and he was classified as "Failure" according to the RIFLE criteria (Risk, Injury, Failure, Loss, End-stage kidney disease) or "Stage 3" using the Acute Kidney Injury Network (AKIN) criteria.

The patient required several sessions of HD (Fig. 2). The cause of AKI was unknown, and, therefore, we could not make a judgment as to whether his renal function would improve. Chemical renal disturbances or pre-renal hypovolemia were shown not to be the cause of AKI. We, therefore, considered that we needed to carry out enhanced CT to look for the source of infection and renovascular aberrations.

The degree of AKI resulted in acute flare-ups every day, and the patient developed uremic symptoms and, finally, fluid accumulation. It was, therefore, necessary to perform HD until sufficient renal function was restored.

We also performed a kidney biopsy to ascertain the cause of renal failure and obtained 19 glomeruli, none of which showed sclerotic or crescentic changes. While we did not observe any changes in the glomeruli (Fig. 3a), edema was present in the tubulointerstitial area (Fig. 3b). When the patient's renal insufficiency recovered, his serological findings normalized, whereas his UA level remained extremely low. His mother informed us that his father also had hypouricemia (UA $2.0 \mathrm{mg} / \mathrm{dL}$ ), detected in a previous annual medical examination. We, therefore, considered that the patient had familial hypouricemia. During the period of renal dysfunction, his UA level was relatively lower than either the UN or Cre levels.

The UA dynamics of the patient included an extremely low serum UA level $(0.6 \mathrm{mg} / \mathrm{dL})$ and marked increases in daily urinary UA excretion and UA clearance (Cua) (daily urinary UA excretion $760 \mathrm{mg} /$ day, normal limit
Table 1 Blood analysis and urinalysis on admission

\begin{tabular}{|c|c|}
\hline \multicolumn{2}{|l|}{ Urine } \\
\hline Specific gravity & 1.011 \\
\hline $\mathrm{pH}$ & 6.0 \\
\hline Protein & $(1+)$ \\
\hline Sugar & $(-)$ \\
\hline Occult blood & $(1+)$ \\
\hline Urine protein & $0.43 \mathrm{~g} / 24 \mathrm{~h}$ \\
\hline \multicolumn{2}{|l|}{ Blood } \\
\hline Leukocyte count & $9800 / \mu \mathrm{L}$ \\
\hline Erythrocyte count & $379 \times 10^{4} / \mu \mathrm{L}$ \\
\hline Hemoglobin & $12.1 \mathrm{~g} / \mathrm{dL}$ \\
\hline Hematocrit & $33.4 \%$ \\
\hline Platelet count & $16.6 \times 10^{4} / \mathrm{L}$ \\
\hline Urea nitrogen & $90 \mathrm{mg} / \mathrm{dL}$ \\
\hline Cre & $7.6 \mathrm{mg} / \mathrm{dL}$ \\
\hline Uric acid & $5.9 \mathrm{mg} / \mathrm{dL}$ \\
\hline Total protein & $6.8 \mathrm{~g} / \mathrm{dL}$ \\
\hline Albumin & $4.4 \mathrm{~g} / \mathrm{dL}$ \\
\hline Sodium & $133 \mathrm{mEq} / \mathrm{L}$ \\
\hline Potassium & $4.3 \mathrm{mEq} / \mathrm{L}$ \\
\hline Chloride & $96 \mathrm{mEq} / \mathrm{L}$ \\
\hline Calcium & $2.29 \mathrm{mmol} / \mathrm{L}$ \\
\hline Phosphate & $1.87 \mathrm{mmol} / \mathrm{L}$ \\
\hline C-reactive protein & $3.31 \mathrm{mg} / \mathrm{dL}$ \\
\hline Creatine kinase & $121 \mathrm{U} / \mathrm{L}$ \\
\hline Myoglobin & $39.5 \mathrm{ng} / \mathrm{mL}($ normal $<60)$ \\
\hline PR3-ANCA & $<10$ EU $($ normal <10) \\
\hline MPO-ANCA & $<10$ EU $($ normal <10) \\
\hline \multicolumn{2}{|c|}{ Arterial blood gas (room air) } \\
\hline $\mathrm{pH}$ & 7.384 \\
\hline $\mathrm{PCO}_{2}$ & $4.59 \mathrm{kPa}(34.6 \mathrm{mmHg})$ \\
\hline $\mathrm{PO}_{2}$ & $13.0 \mathrm{kPa}(97.2 \mathrm{mmHg})$ \\
\hline $\mathrm{HCO}_{3}^{-}$ & $20.1 \mathrm{mmol} / \mathrm{L}$ \\
\hline
\end{tabular}

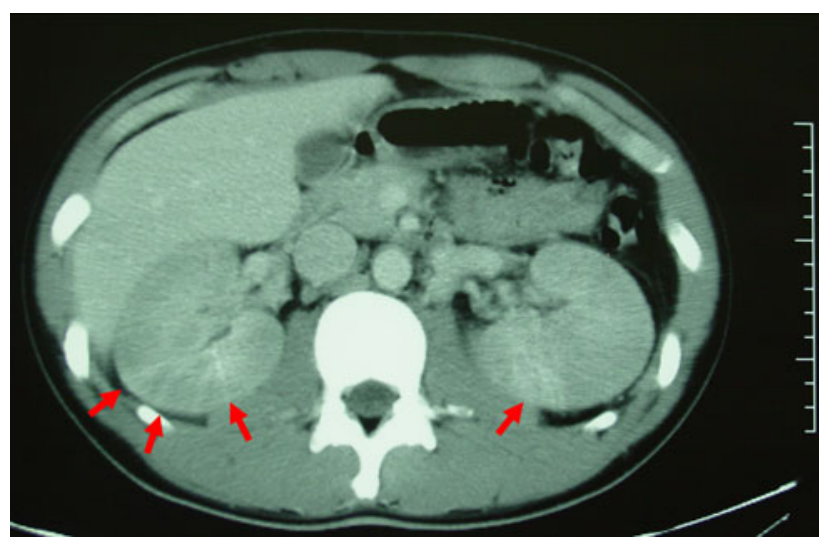

Fig. 1 Enhanced computed tomography (CT) scan image showing enlarged kidneys with weak enhancement. No wedge-shaped contrast media was detected, consistent with the classification of a "patchy" shape (red arrows) 


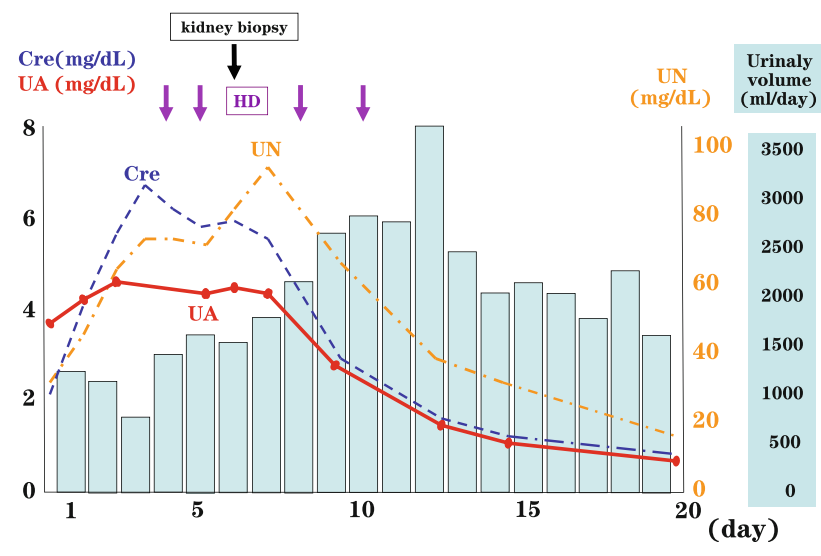

Fig. 2 Clinical course of the patient. The patient developed acute kidney injury (AKI) without oliguria. His serum creatinine (Cre) and urea nitrogen (UN) levels increased rapidly day after day, requiring several hemodialysis (HD) sessions from the fourth disease day. During this period of dialysis, he was not oliguric. A kidney biopsy was performed on the fifth disease day. His urine volume increased after the second dialysis session. Following improvement in his clinical condition, we noticed that his serum uric acid (UA) level was markedly low

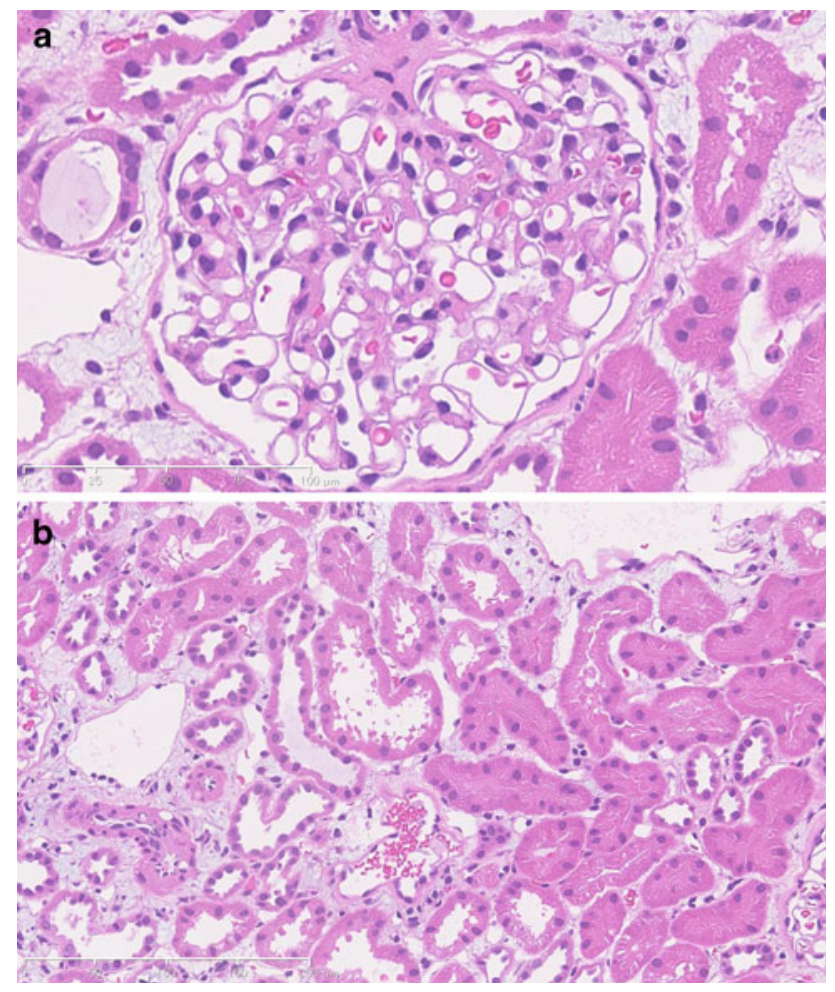

Fig. 3 Kidney biopsy histological findings. a H\&E stain, $\times 400$. The glomerular basal membrane and mesangial area remained unchanged, with normal glomerular findings. b H\&E stain, $\times 200$. Renal tubular atrophy and cellular infiltration to the tubulointerstitial area were not observed. Interstitial edema was apparent in the cortex, although the shape of the renal tubules was maintained

$8.6 \pm 51.7 /$ day; Cua $75.8 \mathrm{~mL} / \mathrm{min} / 1.73 \mathrm{~m}^{2}$, normal limit $7.3 \pm 1.4 \mathrm{~mL} / \mathrm{min})$. Creatinine clearance (Ccr) was normal $\left(108.8 \mathrm{~mL} / \mathrm{min} / 1.73 \mathrm{~m}^{2}\right)$, whereas the $\mathrm{Cua} / \mathrm{Ccr}$ ratio was markedly increased $(70.0 \%$, normal limit $7.3 \pm 1.4 \mathrm{~mL} /$ $\min 7.3 \pm 13)$.

We then performed a pyrazinamide (PZA) loading test. PZA is a drug that causes decreased urate excretion in tubules. The results showed no decrease in the $\mathrm{Cua} / \mathrm{Ccr}$ ratio $(14.3 \%$, normal limit almost $0 \%)$. Gene analysis of the patient showed that he had a G774A mutation that dominated the SLC22A12 gene encoding URAT1.

\section{Discussion}

In this case, a patient with familial renal hypouricemia as a basal disease developed AKI following sudden exercise. This condition is termed ALPE and is induced by anaerobic exercise, leading to renal dysfunction without an increase in either myoglobin or creatine phosphokinase [2-4]. A possible mechanism for the disorder involves renovascular spasm, resulting in the development of ALPE and loin pain. As a result of this spasm, persistent contrast media is found in wedge shapes on enhanced CT images [6]. Approximately $10 \%$ of patients with renal hypouricemia have a past history of ALPE, and, accordingly, hypouricemia is regarded as a risk factor for AKI. UA acts as an antioxidant, and it is thought that it may scavenge a large quantity of free radicals generated by exercise [7]. As the patients with renal hypouricemia had small amounts of UA, the kidney may have suffered severe effects from active oxygen. Spasm of a renal interlobar artery may ultimately occur, and it is thought that this may be a cause of ALPE [6].

Renal hypouricemia is an inherited and heterogeneous disorder characterized by increased urate clearance. UA is secreted and reabsorbed in renal tubules, with the disorder causing hypouricemia. The disease has been classified into four types, a system termed the "four-component hypothesis" [8].

Anti-tuberculous PZA inhibits the secretory phase of the UA transport mechanism in renal tubules by almost completely inhibiting urate excretion [9]. If the UA transporter function is normal, the results of a PZA loading test should result in a decrease in UA excretion to approximately $0 \%$. When the urate excretion is not inhibited by PZA, this is consistent with the presence of the presecretory reabsorptive site type of hypouricemia. In accordance with the fourcomponent model, the results of the PZA loading test in our patient confirmed the diagnosis of a presecretory reabsorptive site. However, based on recent evidence, we believe that it is necessary to reconsider the four-component model hypothesis.

The details of the role of URAT1 have been reported recently [10]. URAT1 is a novel member of the organic anion transporter (OAT) family, which belongs to the 
major facilitator superfamily [11]. URAT1 encoded by $S L C 22 A 12$ is located at the apical membrane in proximal tubules and regulates serum UA concentration. A loss of function of URAT1 causes the development of marked hypouricemia [1,5]. Renal hypouricemia is common in the Japanese population, with the G774A nonsense mutation occurring in about $80 \%$ of cases of renal hypouricemia [5, 12]. We confirmed that the patient in this study had this gene mutation (G774A homozygote).

When our patient's renal function recovered, his serum UA level was markedly low at $0.6-0.9 \mathrm{mg} / \mathrm{dL}$. Patients with a UA level $<2.0 \mathrm{mg} / \mathrm{dL}$ are defined as having hypouricemia. The gene mutation in cases with serum UA levels $<1.0 \mathrm{mg} / \mathrm{dL}$ are classified as homozygotes or compound heterozygotes [5]. The patient's father's had serum UA levels of approximately $2.0 \mathrm{mg} / \mathrm{dL}$, a level that was initially considered to be inconsequential. However, this family history proved to be very important for making a definitive diagnosis in our patient, and emphasizes the importance of obtaining a careful medical history.

Urinary calculi are a frequent complication associated with hypouricemia, although this complication was not observed in our patient. The mechanism leading to this complication is thought to involve hypouricemia causing increased UA excretion in the urine.

There is no particular therapy for renal hypouricemia, with limiting excessive exercise being recommended for preventing ALPE. When our patient developed AKI, his serum UA level remained within normal limits, with the increase in levels being relatively lower than the increase in Cre and UN levels. During the period of AKI, his UA level, therefore, was within normal limits. This indicated that, in patients with AKI and normal levels of UA, it is important to consider the possibility of the coexistence of renal hypouricemia.

We performed a kidney biopsy during the acute phase of AKI in our patient, and carried out a histological examination. There are only a small number of reports of kidney biopsy in similar cases. In early reports, the deposition of UA was found in kidney tissue [13], whereas later reports showed renal tubular necrosis, with the assumption that glomeruli had normal histology [14-17]. In our case, interstitial edema was apparent, although the shape of the renal tubules was maintained and there were no changes observed in the glomeruli. However, it remains unclear whether this interstitial edema was the sole cause of the vasospasm in our patient. When evaluating the findings of renal biopsy tissue of ALPE caused by hypouricemia, we consider that it is also useful to determine the mechanism leading to these changes. We consider it to be possible that the accrual of interstitial edema not only causes vasospasm, but it may also increase capillary permeability. However, the association between hypouricemia and hypoxic conditions remains uncertain in the pathogenesis of tubulointerstitial damage.

The details of the mechanism of how renal hypouricemia resulted in ALPE are not clear. However, enhanced CT of these cases shows that their kidneys have pathognomonic findings. In the early phase, contrast media images showed a wedge shape loss. In contrast, it is known that this imaging effect remains for $48 \mathrm{~h}$ within the region in which it was missing during the early phase [6].

A hypothesis to explain this phenomenon requires the acceptance that renal interlobar artery spasm is caused by anaerobic exercise [6]. Remarkably, the renal biopsy tissue showed interstitial edema between the proximal renal tubules. However, there were no findings of collapse such as wrinkling in the glomerular basement membrane. We, therefore, formulated the following hypothesis. The "patchy" shape defect seen in the image of ALPE indicated massive tubulointerstitial edema, which may have led to post-glomerular ischemia. Post-glomerular ischemia caused by a decrease in renal blood flow in a peritubular capillary needed to be excluded, as it was considered to be more important clinically than pre-glomerular ischemia caused by renal artery spasm.

There are numerous reports on gene mutations associated with renal hypouricemia. The gene mutation in the patient is the most common type found in the Japanese population. Papers reporting both AKI which underwent dialysis therapy and kidney biopsy tissue are rare and, therefore, we consider that our case provides additional valuable information.

In conclusion, we treated a case of ALPE caused by renal hypouricemia who required dialytic therapy. Gene analysis showed a nonsense mutation of G774A in the SLC22A12 gene. This mutation is a common genetic type in the Japanese population. In this case, the kidney biopsy histology showed only interstitial edema.

Acknowledgments We thank Kimiyoshi Ichida MD, PhD (Department of Pathophysiology, Tokyo University of Pharmacy and Life Sciences) for performing the gene analyses.

\section{References}

1. Enomoto A, Kimura H, Chairoungdua A, Shigeta Y, Jutabha P, Cha $\mathrm{SH}$, et al. Molecular identification of a renal urate anion exchanger that regulates blood urate levels. Nature. 2002;417:447-52.

2. Ishikawa I, Onouchi Z, Yuri T, Saito Y, Shinoda A, Yamamoto I. Acute renal failure with severe loin pain and patchy renal vasoconstriction. In: Eliahou HE, editor. Acute renal failure. London: John Libbey; 1982. p. 224-9.

3. Ishikawa I. Acute renal failure with severe loin pain and patchy renal ischemia after anaerobic exercise in patients with or without renal hypouricemia. Nephron. 2002;91:559-70.

4. Oh KJ, Lee HH, Lee JS, Chung W, Lee JH, Kim SH, et al. Reversible renal vasoconstriction in a patient with acute renal failure after exercise. Clin Nephrol. 2006;66:297-301. 
5. Ichida K, Hosoyamada M, Hisatome I, Enomoto A, Hikita M, Endou $\mathrm{H}$, et al. Clinical and molecular analysis of patients with renal hypouricemia in Japan-influence of URAT1 gene on urinary urate excretion. J Am Soc Nephrol. 2004;15:164-73.

6. Ishikawa I, Saito Y, Shinoda A, Onouchi Z. Evidence for patchy renal vasoconstriction in man: observation by CT scan. Nephron. 1981;27:31-4.

7. Murakami T, Kawakami H, Fukuda M, Shiigi H. Recurrence of acute renal failure and renal hypouricaemia. Pediatr Nephrol. 1993;7:772-3.

8. Levinson DJ, Sorensen LB. Renal handling of uric acid in normal and gouty subject: evidence for a 4-component system. Ann Rheum Dis. 1980;39:173-9.

9. Garcia Puig J, Mateos Antón F, Muñoz Sanz A, Gaspar G, Lesmes A, Ramos T, et al. Renal handling of uric acid in normal subjects by means of the pyrazinamide and probenecid tests. Nephron. 1983;35:183-6.

10. Guggino SE, Aronson PS. Paradoxical effects of pyrazinoate and nicotinate on urate transport in dog renal microvillus membranes. J Clin Invest. 1985;76:543-7.

11. Enomoto A, Endou H. Roles of organic anion transporters (OATs) and a urate transporter (URAT1) in the pathophysiology of human disease. Clin Exp Nephrol. 2005;9:195-205.
12. Iwai N, Mino Y, Hosoyamada M, Tago N, Kokubo Y, Endou H. A high prevalence of renal hypouricemia caused by inactive SLC22A12 in Japanese. Kidney Int. 2004;66:935-44.

13. Erley CM, Hirschberg RR, Hoefer W, Schaefer K. Acute renal failure due to uric acid nephropathy in a patient with renal hypouricemia. Klin Wochenschr. 1989;67:308-12.

14. Ito O, Hasegawa Y, Sato K, Mitsui H, Yuda F, Sato H, et al. A case of exercise-induced acute renal failure in a patient with idiopathic renal hypouricemia developed during antihypertensive therapy with losartan and trichlormethiazide. Hypertens Res. 2003;26:509-13.

15. Ohta T, Sakano T, Ogawa T, Kato J, Awaya Y, Kihara H, et al. Exercise-induced acute renal failure with renal hypouricemia: a case report and a review of the literature. Clin Nephrol. 2002;58:313-6.

16. Watanabe T, Abe T, Oda Y. Exercise-induced acute renal failure in a patient with renal hypouricemia. Pediatr Nephrol. 2000;14:851-2.

17. Kikuchi Y, Koga H, Yasutomo Y, Kawabata Y, Shimizu E, Naruse M, et al. Patients with renal hypouricemia with exerciseinduced acute renal failure and chronic renal dysfunction. Clin Nephrol. 2000;53:467-72. 